\title{
Gastro-Highlights 2008
}

Mühlhaupt, B ; Schöfl, R ; Rösch, T ; Bauerfeind, P ; Schwizer, W ; Wirth, Hans-Peter ; Rogler, G ; Fried, M

DOI: https://doi.org/10.1007/s11377-008-0255-1

Posted at the Zurich Open Repository and Archive, University of Zurich ZORA URL: https://doi.org/10.5167/uzh-155889

Journal Article

Published Version

Originally published at:

Mühlhaupt, B; Schöfl, R; Rösch, T; Bauerfeind, P; Schwizer, W; Wirth, Hans-Peter; Rogler, G; Fried, M (2009). Gastro-Highlights 2008. Der Gastroenterologe, 4(1):74-77.

DOI: https://doi.org/10.1007/s11377-008-0255-1 
Gastroenterologe 2009 4 4:74-77 DOI 10.1007/s11377-008-0255-1

Online publiziert: 8. Januar 2009

c) Springer Medizin Verlag 2008

\author{
B. Mühlhaupt ${ }^{1} \cdot$ R. Schöfl ${ }^{2} \cdot$ T. Rösch ${ }^{3} \cdot$ P. Bauerfeind ${ }^{1} \cdot$ W. Schwizer ${ }^{1} \cdot$ H.-P. Wirth ${ }^{1}$. \\ G. Rogler ${ }^{1} \cdot$ M. Fried ${ }^{1}$ \\ ${ }^{1}$ Klinik für Gastroenterologie und Hepatologie, Universitätsspital Zürich \\ ${ }^{2}$ Gastroenterologie/Hepatologie, Krankenhaus der Elisabethinen, Linz \\ ${ }^{3}$ Klinik für interdisziplinäre Endoskopie, Universitätsklinikum Hamburg-Eppendorf
}

\title{
Gastro-Highlights 2008
}

\section{Hepatologie}

Nach den Resultaten einer US-amerikanischen Studie zur Wirksamkeit der zugelassenen antiviralen Therapieregimes, an der 3070 Patienten mit einer chronischen Hepatitis C vom Genotyp 1 teilnahmen, führte die Behandlung mit wöchentlich $180 \mu \mathrm{g}$ pegyliertem Interferon(IFN-) $\alpha_{2 \mathrm{a}}$ und täglich 1000-1200 mg Ribavirin nach 48 Wochen bei einem ähnlichen Anteil der Patienten zu einer dauerhaften Viruselimination wie die Therapie mit wöchentlich 1,0 oder $1,5 \mu \mathrm{g}$ pegyliertem IFN- $\alpha_{2 b}$ pro Kilogramm Körpergewicht und täglich 800-1400 mg Ribavirin [1]. In einer prospektiven Studie mit 2312 Patienten mit einer chronischen Hepatitis $\mathrm{C}$, die auf eine vorherige antivirale Therapie nicht angesprochen oder einen Rückfall erlitten hatten, wurde unter der 48-wöchigen Therapie mit pegyliertem IFN- $\alpha$ und Ribavirin in rund einem Viertel der Fälle eine anhaltende Viruselimination erreicht. Dabei wurden die höchsten Ansprechraten bei den Patienten verzeichnet, die an einer Infektion vom Genotyp 2 oder 3 litten und deren Viruskonzentration nach 12 Behandlungswochen unter die Nachweisgrenze abgefallen war [2].

Erste Resultate einer US-amerikanischen Studie, die 74 Patienten mit einer durch chronische Hepatitis C hervorgerufenen Leberzirrhose und Thrombozytopenie umfasste, weisen darauf hin, dass die Behandlung mit dem oralen Thrombopoietin-Rezeptoragonisten Eltrombopag einen deutlichen Anstieg der Thrombozytenzahl bewirkte und damit den Beginn bzw. die Fortsetzung der antiviralen The- rapie ermöglichte [3]. Eine Verkürzung der Behandlung der Hepatitis-C-Infektion vom Genotyp 1 könnte mit dem Proteaseinhibitor Telaprevir erreicht werden, dessen zusätzlich zu pegyliertem IFN- $\alpha_{2 a}$ und Ribavirin erfolgende Verabreichung während der ersten 12 Wochen der insgesamt 24-wöchigen Behandlung bei einem wesentlich größeren Anteil der Patienten $\mathrm{zu}$ einer dauerhaften Viruselimination führte und überdies mit einer geringeren Rückfallrate einherging als die 48-wöchige Standardtherapie mit pegyliertem IFN- $\alpha_{2 a}$ und Ribavirin [4].

Als wirksame Substanz zur Behandlung der chronischen Hepatitis B hat sich das Nukleosid Tenofovir erwiesen, das sowohl bei HBeAg-positiven als auch bei HBeAgnegativen Patienten nach 48 Wochen eine signifikant höhere Ansprechrate bezüglich Viruselimination ergab als die Therapie mit Adefovir und selbst nach einer Behandlungsdauer von 72 Wochen keine Resistenzen hervorrief $[5,6]$. Zum Langzeitverlauf der chronischen Hepatitis B zeigte eine über 12,5 Jahre durchgeführte prospektive Kohortenstudie aus Taiwan, dass die Gesamtmortalität und die leberspezifische Mortalität bei den HBsAg-positiven Patienten mit steigenden HBVDNA-Spiegeln zunahm und im Vergleich zu den HBsAg-negativen Personen signifikant erhöht war [7].

\section{Pankreas}

Nach einer US-amerikanischen, auf Evidenz beruhenden Studie können verschiedene gängige Wirkstoffe eine akute Pankreatitis auslösen, weshalb bei Patienten mit einer akuten Pankreatitis im- mer auch die verwendeten Medikamente überprüft werden sollten [8]. Die chronische Dialysebehandlung stellt nach einer Erhebung in deutschen Dialysezentren einen Risikofaktor für die Entwicklung einer akuten Pankreatitis dar, wobei die Entzündung bei den Peritonealdialysepatienten mit einer höheren Inzidenz auftritt und überdies häufiger einen nekrotisierenden Verlauf nimmt als bei den Hämodialysepatienten [9]. Auf die prophylaktische Verwendung von Probiotika sollte im Falle einer akuten Pankreatitis verzichtet werden, nachdem in einer doppelblinden Studie mit 298 Patienten mit einem Verdacht auf eine schwere akute Pankreatitis bei der Behandlung mit einer sechs unterschiedliche Bakterienstämme enthaltenden Zubereitung im Vergleich zu Plazebo keine Reduktion des Infektionsrisikos, aber ein Anstieg der Mortalität festgestellt wurde [10].

\section{(7) Bei akuter Pankreatitis sollten immer auch die verwendeten Medikamente überprüft werden}

Die aus tierexperimentellen Untersuchungen vermutete antikanzerogene Wirkung der Statine konnte in einer retrospektiven Fallkontrollstudie anhand der Daten von 483.733 Personen der Veterans-Affairs-Datenbank bestätigt werden, in der das Risiko für ein Pankreaskarzinom bei der Anwendung von Statinen über mindestens 6 Monate um $67 \%$ und bei einer Statinanwendung über mindestens 4 Jahre sogar um 80\% verringert wurde [11]. Demgegenüber bewirkte ein hoher Gemüsekonsum in der multieth- 
nischen Kohortenstudie „Hawaii-Los Angeles" mit 183.522 Teilnehmern nach einer Nachbeobachtungsdauer von 8,3 Jahren nur bei den Personen mit einem hohen Karzinomrisiko wie den Rauchern, den Afroamerikanern und den Übergewichtigen eine Reduktion des Risikos für ein Pankreaskarzinom [12].

Gemäß einer Metaanalyse von 20 Studien mit 6296 Patienten mit einem inoperablen Pankreaskarzinom führte die Verabreichung von Gemcitabin in Kombination mit einem weiteren Zytostatikum oder einem zielgerichteten molekularen Wirkstoff gegenüber der alleinigen Behandlung mit Gemcitabin zu einer signifikanten, aber nur geringfügigen Verbesserung der Ansprechrate und der progressionsfreien Überlebenszeit, ohne jedoch die Gesamtüberlebenszeit zu verlängern, weshalb die Monotherapie mit Gemcitabin weiterhin als Standardtherapie des fortgeschrittenen Pankreaskarzinoms gilt [13].

\section{Gastrointestinale Endoskopie}

Bezüglich der diagnostischen Zuverlässigkeit des Narrowband-Imaging zeigte eine prospektive Studie mit 65 Patienten mit einem dysplastischen Barrett-Ösophagus, dass mit diesem Untersuchungsverfahren bei einem signifikant größeren Anteil der Patienten Dysplasien und vor allem höhergradige Dysplasien bei einer wesentlich geringeren Anzahl entnommener Biopsien entdeckt werden als mit der konventionellen Endoskopie [14]. Bei der Beurteilung von 22 Arealen eines BarrettÖsophagus durch 7 Endoskopiker ergab die konventionelle Endoskopie bezüglich des Nachweises von frühen Neoplasien eine Treffsicherheit von $86 \%$, die weder durch die Färbung mit Indigokarmin oder Essigsäure noch durch das NarrowbandImaging erhöht werden konnte [15].

Die in einer europäischen Multizenterstudie bei 151 Patienten mit einem neoplastischen Barrett-Ösophagus untersuchte stufenweise radikale endoskopische Resektion ermöglichte in $99 \%$ der Fälle eine vollständige Eradikation der Neoplasie und in 97\% der Fälle eine komplette Entfernung der intestinalen Metaplasie, wobei nach einer Nachbeobachtungsdauer von 18 Monaten 3\% der Pati- enten eine rezidivierende Neoplasie und 19\% der Patienten ein Rezidiv des BarrettÖsophagus entwickelten [16]. Außerdem wurde in einer Studie mit 31 Patienten mit einem neoplastischen Barrett-Ösophagus durch die kombinierte Anwendung der endoskopischen Resektion und der Radiofrequenzablation bei 97\% der Patienten eine komplette Remission der Dysplasie und des Barrett-Ösophagus erreicht, und nach einer Nachbeobachtungsdauer von 12 Monaten wurde lediglich bei einem Patienten ein Rezidiv des Barrett-Ösophagus festgestellt [17].

Als Weiterentwicklung der endoskopischen Antirefluxtherapie steht seit kurzem das transorale inzisionslose Fundoplikationssystem „EsophyX“ zur Verfügung, das in einer prospektiven Multizenterstudie mit 86 Patienten mit einer chronischen gastroösophagealen Refluxkrankheit nach einer Nachbeobachtungsdauer von 12 Monaten zu einer deutlichen Verbesserung der ösophagealen Säurekontrolle führte und damit bei ungefähr der Hälfte der Patienten das Absetzen der medikamentösen Säuresuppression erlaubte [18]. Des Weiteren bewirkte auch die endoskopische Implantation des Kunststoffmaterials „Enteryx“ in den unteren Ösophagussphinkter in einer Multizenterstudie mit 85 an Sodbrennen leidenden Patienten nach einer Nachbeobachtungsdauer von 6 Monaten eine signifikante Verbesserung der ösophagealen Säurekontrolle, so dass rund 3 Viertel der Patienten auf die antisekretorische Therapie verzichten konnten [19].

\section{Kolorektales Karzinom}

Zur Wirksamkeit der adjuvanten Chemotherapie des kolorektalen Karzinoms zeigte eine englische Multizenterstudie mit 3239 Patienten, dass die Überlebensrate durch die im Anschluss an die kurative Resektion erfolgende Chemotherapie mit 5-Fluorouracil und Leucovorin im Vergleich zur alleinigen Nachbeobachtung nach 5,5 Jahren um 3,6\% erhöht wurde [20]. Die zusätzlich zur Resektion vorgenommene perioperative Chemotherapie mit Oxaliplatin, 5-Fluorouracil und Leucovorin führte in einer französischen Multizenterstudie mit 364 Patienten mit Lebermetastasen infolge eines kolorek- talen Karzinoms gegenüber der alleinigen chirurgischen Therapie nach einer Nachbeobachtungsdauer von 3 Jahren zu einer Erhöhung der progressionsfreien Überlebensrate um 8,1\% [21]. Außerdem bewirkte in einer kanadischen Studie auch die Behandlung mit dem monoklonalen Antikörper Cetuximab bei den 572 Patienten mit einem kolorektalen Karzinom, die auf eine vorangegangene palliative Chemotherapie nur ungenügend angesprochen hatten, im Vergleich zu den rein palliativen Maßnahmen eine geringfügige Verlängerung der medianen Überlebenszeit von 4,6 auf 6,1 Monate [22].

\section{- Der Stellenwert der Koloskopie als Goldstandard bei der Karzinomprävention wird durch die Resultate einer US- amerikanischen Studie gestützt.}

In dieser retrospektiven Studie mit 6283 Teilnehmern wurden mit dem konventionellen Verfahren fortgeschrittene Neoplasien mit einer ähnlichen Treffsicherheit nachgewiesen wie mit der CTKolonographie. Allerdings wurden bei den mit der konventionellen Koloskopie untersuchten Personen nahezu alle Polypen entfernt, während bei der virtuellen Koloskopie nur gerade 8\% der Patienten einer Polypektomie zugeführt wurden, was mit einer Kostenreduktion einherging [23].

Dies wirft die Frage auf, ob wirklich alle Polypen abgetragen und histologisch aufgearbeitet werden müssen. In diesem $\mathrm{Zu}$ sammenhang zeigte eine weitere Studie, dass bei 4474 konsekutiven Koloskopien 9042 von insgesamt 10.400 entfernten Polypen eine Größe unter $6 \mathrm{~mm}$ aufwiesen. Da es sich bei Polypen mit einer Größe unter $6 \mathrm{~mm}$ nur in weniger als o, $1 \%$ der Fälle um Karzinome handelt, könnte mit einer Screeningstrategie, bei der alle Polypen abgetragen werden, aber nur bei Polypen mit einer Größe über $6 \mathrm{~mm}$ eine histologische Untersuchung durchgeführt wird, erhebliche Kosteneinsparungen erzielt werden [24].

\section{Ösophaguserkrankungen}

Zur Bedeutung der ösophagealen Säureexposition bei der Entstehung der Re- 
fluxsymptome ergab eine englische Studie, dass die Infusion von Salzsäure in die Speiseröhre bei den 14 Patienten mit einer nichterosiven Refluxkrankheit stärkere Schmerzen hervorrief als bei den 14 Patienten mit einer Refluxösophagitis und den 12 gesunden Kontrollpersonen. Diese ösophageale Hypersensibilität war bei den 11 Patienten mit funktionellem Magenbrennen noch ausgeprägter, da sowohl die Infusion von Salzsäure als auch von Natriumchlorid Symptome auslöste [25]. Außerdem war in einer japanischen Studie die sekundäre Peristaltik bei den 2o Patienten mit einer nichterosiven Refluxkrankheit deutlich geringer als bei den 20 gesunden Kontrollpersonen, weshalb auch eine verlängerte Säurekontaktzeit zur Symptomentstehung beitragen könnte [26]. Im Rahmen einer französischen Studie wurde bei den 20 Patienten, die trotz einer Behandlung mit einem hochdosierten Protonenpumpeninhibitor an Refluxsymptomen litten, während der symptomatischen Refluxepisoden signifikant häufiger ein bis in den proximalen Ösophagus gelangender Reflux festgestellt als während der asymptomatischen Refluxepisoden [27].

Nachdem mehrere klinische Studien einen Zusammenhang zwischen dem Übergewicht und dem Adenokarzinom des Ösophagus ergaben $[28,29]$, stellt sich die Frage, inwieweit die Entstehung des Barrett-Ösophagus als einzigem bekannten Risikofaktor für das Ösophaguskarzinom durch das Übergewicht begünstigt wird. Dabei zeigen die Resultate einer englischen Metaanalyse, dass ein hoher Körpermassenindex die Wahrscheinlichkeit für die Progression einer Refluxkrankheit zu einem Barrett-Ösophagus nicht erhöht [3o]. Demgegenüber erwies sich in einer US-amerikanischen Fallkontrollstudie mit 193 Patienten mit einem neu diagnostizierten Barrett-Ösophagus die zentrale Adipositas als Risikofaktor für die Entwicklung eines Barrett-Ösophagus, da mit zunehmendem Taillen-Hüft-Verhältnis eine signifikante Erhöhung der Wahrscheinlichkeit für ein spezialisiertes metaplastisches Epithel, einen sichtbaren Barrett-Ösophagus und insbesondere für einen Barrett-Ösophagus mit einem langen Segment festgestellt wurde [31]. Des Weiteren litten in einer irländischen Studie von 192 Patienten mit einem Barrett-Ösophagus $46 \%$ an einem metabolischen Syndrom und $78 \%$ an einer zentralen Adipositas, wobei die Anteile der Patienten mit einem metabolischen Syndrom oder einer zentralen Adipositas in der Gruppe mit einem langen Barrett-Segment signifikant größer waren als in der Gruppe mit einem kurzen Barrett-Segment [32].

\section{Ulkuserkrankungen}

Bezüglich der Auswirkungen der Säuresuppression auf das Infektionsrisiko ergaben eine Fallkontrollstudie und zwei Metaanalysen, dass unter einer Therapie mit einem Protonenpumpeninhibitor ein signifikant erhöhtes Risiko für eine durch Salmonella, Campylobacter, Clostridium difficile und andere Bakterien hervorgerufene Gastroenteritis besteht, wogegen für eine Behandlung mit einem $\mathrm{H}_{2}$-Rezeptor-Antagonisten keine oder höchstens eine minimale Zunahme des Infektionsrisikos festgestellt wurde $[33,34]$. Außerdem führte die Säuresuppression mit einem Protonenpunpeninhibitor, nicht aber mit einem $\mathrm{H}_{2}$-Rezeptor-Antagonisten in einer dänischen Fallkontrollstudie zu einem signifikanten Anstieg des Risikos für eine ambulant erworbene Pneumonie [35]. Zum Einfluss der Säuresuppression auf das Krebsrisiko zeigen die Resultate einer britischen und einer dänischen Fallkontrollstudie, dass eine Langzeittherapie mit einem Protonenpumpeninhibitor keine Zunahme des Risikos für ein kolorektales Karzinom bewirkt [36, 37]. Im Rahmen einer französischen Doppelblindstudie mit 124 Patienten, die nach einer koronaren Stentimplantation mit Aspirin und Clopidogrel behandelt wurden, führte die gleichzeitige 7-tägige Behandlung mit Omeprazol gegenüber Plazebo zu einer signifikanten Reduktion der Wirksamkeit der thrombozytenhemmenden Therapie [38].

Zur gastrointestinalen Verträglichkeit der antirheumatischen Therapie ergaben eine spanische Fallkontrollstudie und eine kanadische Metaanalyse für die Behandlung mit einem selektiven Cyclooxygenase-2-Inhibitor im Vergleich zu einem nichtsteroidalen Antirheumatikum ein verringertes Risiko für obere gastrointestinale Komplikationen, wobei dieser the- rapeutische Vorteil aber bei der gleichzeitigen Anwendung von Aspirin nicht mehr feststellbar war [39, 40]. In einer Doppelblindstudie mit 273 Arthritispatienten mit einer durch nichtsteroidale Antirheumatika hervorgerufenen Ulkusblutung in der Anamnese kam es unter der 12-monatigen Therapie mit Celecoxib und Esomeprazol in keinem einzigen Fall zu rezidivierenden Ulkusblutungen, während bei $8,9 \%$ der mit Celecoxib und Plazebo behandelten Patienten Rezidivblutungen auftraten [41]. Gemäß einer US-amerikanischen Doppelblindstudie mit 854 Arthritispatienten, die zur Kardioprävention mit niedrig dosiertem Aspirin behandelt wurden, führte die Therapie mit Celecoxib nach einer Nachbeobachtungsdauer von 12 Wochen bei einem ähnlichen Anteil der Patienten zu einem gastrointestinalen Ulkus wie die Behandlung mit Naproxen und Lansoprazol [42].

\section{Morbus Crohn}

Nach den Resultaten einer Multizenterstudie mit 133 Patienten mit einem neu diagnostizierten $\mathrm{M}$. Crohn, bei denen unter einer immunsuppressiven Kombinationstherapie mit Infliximab und Azathioprin oder einer konventionellen Behandlung mit Kortikosteroiden nach zwei Jahren eine vollständige Mukosaheilung erzielt wurde, blieb die Remission in den nächsten zwei Jahren bei einem signifikant gröBeren Anteil erhalten als bei den Patienten ohne Mukosaheilung [43]. Die Erhaltungstherapie mit Infliximab führte in einer Studie mit 614 Patienten mit einem M. Crohn nach einer medianen Nachbeobachtungsdauer von 59 Monaten bei $40,3 \%$ der Patienten zu einer vollständigen Mukosaheilung und bei weiteren 20,9\% der Patienten zu einer partiellen Mukosaheilung, wobei der Anteil der Patienten mit einer Mukosaheilung bei der Erhaltungstherapie mit wiederholter Verabreichung von Infliximab signifikant gröBer war als bei der Behandlung mit episodischer Wirkstoffverabreichung [44].

Im Zusammenhang mit den Diskussionen um die Risiken der Biologika zeigte eine Metaanalyse von 21 randomisierten Studien mit 5356 an M. Crohn leidenden Patienten, dass das Risiko für schwere Infektionen und Malignome wie auch die 
Mortalität unter einer Therapie mit einem gegen den Tumornekrosefaktor a gerichteten Wirkstoff im Vergleich zu Plazebo nicht erhöht waren [45]. Des Weiteren traten bei den 1313 M.-Crohn-Patienten, die innerhalb des „Certolizumab Pegol Development Program“ mit pegyliertem Certolizumab behandelt wurden, Lymphome, Herzinsuffizienz, Tuberkulose oder Todesfälle mit einer ähnlichen Häufigkeit auf wie bei den Patienten der Plazebogruppe [46]. Nach den kombinierten Daten von mehreren klinischen Studien führte die Behandlung mit Adalimumab im Vergleich zur Standardtherapie nicht nur bei einem größeren Anteil der Patienten zu einem Fistelverschluss und einer länger anhaltenden Remission, sondern sie ging überdies mit einer geringeren Inzidenz schwerer Infektionen und Malignome einher [47]. Im Gegensatz zu diesen beruhigenden Daten zeigt eine Metaanalyse von 26 Studien mit 8843 M.-CrohnPatienten, dass das Risiko für ein NonHodgkin-Lymphom bei den mit einem Tumornekrosefaktor- $\alpha$-Hemmer behandelten Patienten im Vergleich zur allgemeinen Bevölkerung signifikant um den Faktor 2,88, gegenüber den mit einem Immunomodulator behandelten Patienten jedoch nur tendenziell erhöht war [48].

\title{
Korrespondenzadresse
}

\section{Prof. Dr. M. Fried}

Klinik für Gastroenterologie und Hepatologie, Universitätsspital Zürich

Rämistr. 100, 8091 Zürich, Schweiz

michael.fried@usz.ch

Interessenkonflikt. Der korrespondierende Autor gibt an, dass kein Interessenkonflikt besteht.

\section{Das Literaturverzeichnis ...}

... finden Sie in der html-Version dieses Beitrags im Online-Archiv auf der Zeitschriftenhomepage www.DerGastroenterologe.springer.de

\section{Hier steht eine Anzeige.}

\author{
量 Springer
}

